\title{
Evaluation Activities from the National Syndromic Surveillance Program
}

\author{
Sebastian Romano*1, Cassandra Davis ${ }^{1}$, Krystal Collier², Sara Johnston², \\ Hana Tesfamichael ${ }^{1}$ and Hussain Yusuf ${ }^{1}$
}

${ }^{1}$ Centers for Disease Control and Prevention, Atlanta, GA, USA; ${ }^{2}$ Arizona Department of Health Services, Phoenix, AZ, USA

\section{Objective}

The objective of this session is to discuss syndromic surveillance evaluation activities. Panel participants will describe contexts and importance of selected evaluation and performance measurement activities in NSSP. Discussions will explore ways to strengthen evaluation in syndromic surveillance activities in the future.

\section{Introduction}

Syndromic surveillance uses near-real-time Emergency Department healthcare and other data to improve situational awareness and inform activities implemented in response to public health concerns. The National Syndromic Surveillance Program (NSSP) is a collaboration among state and local health departments, the Centers for Disease Control and Prevention (CDC), other federal organizations, and other entities, to strengthen the means for and the practice of syndromic surveillance. NSSP thus strives to strengthen syndromic surveillance at the national and the state, and local levels through the coordinated activities of the involved partners and the development and use of advanced technologies, such as the BioSense platform. Evaluation and performance measurement are crucial to ensure that the various strategies and activities implemented to strengthen syndromic surveillance capacity and practice are effective. Evaluation activities will be discussed at this session and feedback from audience will be sought with the goal to further strengthen evaluation activities in the future.

\section{Keywords}

Syndromic surveillance; Public health practice; Evaluation; Performance measurement

\section{*Sebastian Romano}

E-mail:wwj5@cdc.gov 